\title{
ALOCAÇÃO DE RECURSOS PARA CUIDAR DE IDOSOS DURANTE A PANDEMIA: UMA REFLEXÃO BIOÉTICA
}

\author{
Valéria Pagotto' \\ ORCID: 0000-0002-5590-2453
}

Cintia Maria Tanure Bacelar Antunes'

ORCID: 0000-0001-6699-7681

Gabriela Cristina Cantisani Padua" ORCID: 0000-0003-4473-4586

Rosmari Wittmann-Vieira"'I

ORCID: 0000-0002-7347-1327

Maria Márcia Bachion'

ORCID: 0000-0001-5044-6148

Cristiane José Borges ${ }^{\text {IV }}$

ORCID: 0000-0002-5846-0750

'Universidade Federal de Goiás. Goiânia, Goiás Brasil.

"Universidade de Brasília. Brasília, Distrito Federal, Brasil.

"'Hospital de Clínicas de Porto Alegre. Departamento de Gerontologia. ABEn-RS. Porto Alegre, Rio Grande do Sul, Brasil.

'vUniversidade Federal de Jataí. Jataí, Goiás, Brasil.

Autor Correspondente: Valéria Pagotto

E-mail: valeriapagotto@ufg.br

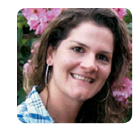

Como citar:

Pagotto V, Antunes CMTB, Padua GCC, Vieira RW

Bachion MM, Borges CJ. Alocação de recursos para cuidar de idosos durante a pandemia: uma reflexão bioética. In: Santana RF (Org.). Enfermagem gerontológica no cuidado do idoso em tempos da COVID 19. Brasilia, DF: Editora ABen; 2021. 171 p. (Serie Enfermagem e Pandemias, 5). https://doi.org/10.51234/aben.21.e05.c06

\section{INTRODUÇÃO}

A pandemia de corona vírus disease 19 (COVID-19) provocada pelo novo vírus SARS-CoV-2 impactou, de forma direta e indireta, em curto período de tempo, os serviços de saúde, ao redor do mundo, que se reorganizaram, buscando soluções rápidas para responder às demandas advindas diante desse novo cenário ${ }^{(1-2)}$.

Nesse contexto, a alocação de recursos para o cuidar em saúde é uma problemática que impõe reflexões bioéticas de modo ampliado ${ }^{(3)}$. Tradicionalmente, o tema alocação de recursos em saúde se detém no exame de acesso a serviços, equipamentos, insumos e profissionais em instituições de saúde ${ }^{(4)}$. Contudo, é necessário ampliar essa visão para considerar a qualidade dos cuidados e o acesso à profissionais adequadamente preparados, em todos os níveis de atenção à saúde e contextos de cuidado.

O Brasil, reconhecido como um dos países com os maiores índices de concentração de renda do mundo ${ }^{(5)}$, atravessa uma crise econômica e política, mesmo antes da pandemia, que impacta diretamente na alocação de recursos, nos diferentes cenários de cuidado, desde o institucional até o domiciliar. Este problema envolve desde itens básicos como o uso de equipamentos básicos de proteção individual, como máscaras, até recursos avançados como leitos de terapia intensiva ${ }^{(3)}$.

Especificamente, entre os idosos, a alocação de recursos durante a pandemia pode ser um desafio ainda maior, pois trata-se do grupo com maior risco de desenvolver complicações graves da doença ${ }^{(6)}$, demandando recursos de diversas complexidades e níveis de atenção. Em todos os ambientes de cuidado, para idosos com limitações, haverá maior demanda de tempo de cuidados profissionais, especialmente da equipe de enfermagem ${ }^{(7)}$. Além disso, em função das recomendações de cuidado para controle da pandemia, os idosos estão expostos à outros problemas, que envolvem a alocação de recursos humanos para o cuidado, como a descontinuidade de seus tratamento ${ }^{(8)}$. 
Esse cenário mostra que, em uma situação emergencial, o desfecho de saúde das pessoas idosas, dependerá da capacidade de organização dos sistemas de saúde e da alocação adequada dos recursos e da rede de suporte social. Assim, é importante refletir de modo ampliado sobre a alocação de recursos destinados a atender as demandas de cuidados deste grupo vulnerável, visando garantir o respeito aos direitos humanos e à dignidade da pessoa idosa.

\section{OBJETIVO}

Refletir, à luz da bioética, sobre a alocação dos recursos para cuidar de idosos durante a pandemia de COVID-19, e o respeito à dignidade humana.

\section{MÉTODOS}

Trata-se de ensaio teórico de natureza reflexiva, fundamentado na bioética e nas recomendações de órgãos oficiais, dentre elas a Organização Mundial de Saúde (OMS) ${ }^{(1-2,8)}$, para o cuidado do idoso durante a pandemia.

A reflexão bioética foi baseada no modelo teórico da bioética principialista ${ }^{(9)}$. Considerando que os princípios da beneficência, não-maleficência, respeito à autonomia e justiça agem com a premissa de garantir a dignidade das pessoas frente as mudanças decorrentes das evoluções tecnológicas e socioculturais, utilizou-se a comparação entre esses e as recomendações de órgãos oficiais.

A apresentação foi organizada em três categorias temáticas: Vulnerabilidade da pessoa idosa no contexto da pandemia; Alocação de Recursos para o atendimento à pessoa idosa com problemas decorrentes da pandemia; Reflexões bioéticas sobre alocação de recursos em casos extremos, vulnerabilidade e manutenção da dignidade humana, da pessoa idosa, na pandemia.

\section{RESULTADOS}

\section{Vulnerabilidade da pessoa idosa no contexto da pandemia}

O panorama da pandemia no Brasil é bastante complexo devido à diversidade geográfica, econômica e política do país. Embora os primeiros casos tenham acontecido em pessoas de estratos econômicos maiores, rapidamente o vírus atingiu grupos desfavorecidos econômica e socialmente, especialmente populações vulneráveis ${ }^{(8)}$.

A vulnerabilidade compreende uma multiplicidade de fatores, tornando-se indispensável que se tenha um olhar integral para a pessoa idosa ${ }^{(10)}$. Os idosos são considerados um grupo vulnerável à COVID-19 por diferentes razões. Do ponto de vista clínico-epidemiológico, por se tratar de um grupo com carga elevada de doenças, e maior número de limitações nas atividades de diária, esse grupo tem maiores chances de hospitalização e óbito por COVID-19 $9^{(11-12)}$.

No Brasil, uma análise recente do estudo Longitudinal de Saúde do Idoso (ELSI-Brasil) mostrou que cerca de 34 milhões de pessoas com mais de 50 anos de idade têm $\geq 1$ morbidade de risco para COVID-19 grave, com distribuição similar entre as regiões. As morbidades, relacionadas à COVID-19 grave, mais prevalentes nesse grupo foram: as doenças cardiovasculares (56\%), obesidade (39\%), artrite $(21 \%)$ e depressão $(18,5 \%)^{(6)}$.

Soma-se a isso as alterações próprias do envelhecimento. Com o aumento da idade há uma diminuição da resposta do sistema imune, bem como as alterações no sistema respiratório, diminuição da eficiência de trocas gasosas, aumentando a suscetibilidade do idoso às doenças infecciosas ${ }^{(13-14)}$, e portanto, com pior prognóstico para COVID-19.

Já do ponto de vista socioeconômico, os idosos que pertencem à famílias de baixa renda podem ser ainda mais afetados. As populações de baixa renda são as mais vulneráveis à contaminação pelo SARS-CoV-2, 
tendo em vista que utilizam com maior frequência transporte público, residem em domicílios com número maior de moradores, tem acesso limitado ao saneamento básico e à saúde além da dificuldade de manter o isolamento social sem redução significativa da renda ou do próprio emprego ${ }^{(15)}$.

Não obstante, idosos institucionalizados são ainda mais vulneráveis à COVID-19 e representam o principal alvo da doença. A transmissiblidade do vírus em uma ILPI é superior aos demais cenários de cuidado, tanto em razão do maior nível de fragilidade, como limitações que impõe a necessidade constante de cuidados ${ }^{(16)}$.

Nas diversas situações de vulnerabilidade dos idosos, será necessário alocar recursos para o cuidado, desde a vigilância dos casos até a atenção a saúde nos diferentes níveis de atenção, incluindo o ambiente domiciliar. Sendo assim, em todo o território brasileiro estratégias foram desenvolvidas visando diminuir o impacto da pandemia pela COVID-19, que envolvem a alocação de recursos em saúde como: testagem da população, abertura de hospitais de campanha, compra de ventiladores mecânicos, compra de equipamentos de proteção individual (EPIs), ampliação do número de leitos de Unidade de Tratamento Intensivo e contratação de profissionais de saúde(17).

Apesar da alocação de recursos em saúde estar atrelada ao acesso a serviços, equipamentos e insumos em instituições de saúde, no caso dos idosos a qualidade dos cuidados depende ainda da alocação de recursos humanos em diferentes cenários.

No âmbito domiciliar, compete inicialmente, aos familiares e cuidadores o atendimento às necessidades da pessoa idosa ${ }^{(7)}$. Embora o distanciamento social tenha como objetivo proteger as pessoas idosas em situação de vulnerabilidade ${ }^{(1)}$, trouxe alguns desafios para idosos e familiares como a continuidade dos cuidados. A maior parte das consultas nos serviços de saúde foram canceladas, há restrição da interação social, maior risco de violência, abandono e/ou negligência, há dificuldade de acesso às medicações, uma vez que uma das orientações para controle da COVID-19 em idosos é que os medicamentos obtidos na rede pública sejam acessados pelo familiar credenciado(5).

Já no âmbito institucional e profissional, os profissionais da área de saúde ganharam destaque, em particular a enfermagem, que atua nas diferentes frentes de cuidado à população, seja na promoção à saúde, vigilância, atenção especilizada entre outros ${ }^{(7)}$.

Durante a pandemia, foi evidenciada a necessidade do cuidado direcionado às especificidades dos idosos, pois possuem características e peculiaridades próprias, bem como mostrou a diversidade, pluralidade e complexidade do envelhecimento humano. Enfatizando assim, a importância de compreender e incorporar os fundamentos teóricos e práticos da gerontologia, para subsidiar sua tomada de decisão clínica e garantir a proteção deste grupo de risco(7).

Ademais, o contexto pandêmico demonstrou a importância de ressignificar os vínculos com os idosos, incitando os profissionais de enfermagem a agir no sentido de preservar a autonomia e independência dos mesmos $^{(7)}$.

É importante que as políticas de enfrentamento à pandemia considerem as numerosas produções científicas que destacam a vulnerabilidade da população idosa e desenvolvam diretrizes que atendam as reais necessidades desta população ${ }^{(18)}$. Por outro lado, quando se remete ao respeito à vida, surgem demandas como: políticas que possibilitem o fortalecimento da atenção primária em saúde, implementação do monitoramento remoto, a garantia de aquisição dos insumos básicos para sobrevivência humana, oferta de apoio às instituições de Longa Permanência para Idosos, assistência para os que vivem em situação de rua, atenção especial aos idosos que cuidam de idosos e aos trabalhadores informais, bem como garantia de atendimento humanitário e voltado aos cuidados paliativos, quando for necessário(18).

\section{Alocação de Recursos em saúde durante a pandemia: reflexões para cuidar de idosos}

Com o colapso da rede de saúde, provocado pela pandemia, onde o número de leitos disponíveis em unidades de tratamento intensivo se mostrou inferior à demanda, foram propostas árvores de decisão em 
vários países do mundo, com critérios específicos, buscando balizar as decisões das equipes envolvidas na tomada de decisão para a destinação de leitos de UTI ${ }^{(19)}$. Entre os critérios adotados, a previsão do número de anos de vida e com isso de produtividade ou retorno econômico para a sociedade, foi posta como item de seleção de prioridade, aplicando a faixa etária nas decisões de alocação, colocando pessoas mais jovens em posição de vantagem em relação aos idosos ${ }^{(20)}$.

No Brasil ocorreu semelhante busca pela delimitação de protocolos que amparassem os profissionais de saúde nesta difícil decisão. O critério etário foi primeiramente recomendado, sendo revogado na segunda versão do protocolo da Associação de Medicina Intensiva Brasileira ${ }^{(18)}$ para alocação de recursos escassos e em esgotamento, durante a pandemia da COVID-19. Considerou-se que a inclusão deste item poderia ser discriminatório, e decidiu-se pela sua retirada, tomando como base um princípio fundamental da bioética, o da dignidade humana(21).

O adequado gerenciamento desta situação de escassez com implicações na garantia dos insumos e adequado atendimento ao ser humano em sua totalidade, não pode ser realizado apenas por juízo relacionado a questões técnicas cronológicas e biológicas, mas também mediante a efetivação dos direitos humanos por parte dos Estados ${ }^{(21)}$.

Seguindo esse direcionamento a Organização Mundial de Saúde definiu os princípios que devem reger essas ações locais e regionais, que são fundamentados na promoção da igualdade, avaliação da utilidade, priorização daqueles que se encontram em pior situação além dos profissionais de saúde. Estabeleceu também que, devido ao significante impacto desta pandemia nos idosos, é inapropriada a utilização de protocolos que excluam ou não priorizem o grupo mais afetado da população. Portanto o compromisso bioéticocom a dignidade humana e direito à saúde deve orientar as ações de assistência das pessoas idosas ${ }^{(20)}$.

Divulgar amplamente as diretrizes de triagem aos leitos de UTI e demonstrar que as decisões são tomadas de maneira ética e imparcial através de um protocolo transparente, aplicado igualmente a todos os pacientes, facilitando os processos de decisão dos profissionais envolvidos diretamente no cuidado, pode reduzir dúvidas e aumentar a confiança da população idosa, bem como de seus familiares diante desta pandemia ${ }^{(18)}$.

A despeito de todos os esforços empregados, situações de terminalidade da vida podem ocorrer, e os profissionais de saúde envolvidos no cuidado devem realizar recomendações de cuidados paliativos apenas quando os demais esforços para o melhor atendimento em saúde foram empregados sem sucesso. Esse direcionamento, não deve, portanto, substituir a alocação dos recursos necessários, independente da idade do paciente, sendo erroneamente usado em substituição à garantia de cuidado integral e equânime ${ }^{(18-19)}$.

\section{Reflexões bioéticas sobre alocação de recursos em casos extremos, vulnerabilidade e manutenção da dignidade humana na pandemia}

Considerando o impacto da pandemia pela COVID-19, na vida das pessoas, e a demanda de reorganização dos serviços de saúde, percebe-se a necessidade de repensar a tomada de decisão pelos profissionais de saúde, especialmente enfermeiros, que lidam diretamente com a interface entre alocação de recursos emergencial e a garantia da dignidade humana aos idosos, em situação de vulnerabilidade, que procuram os serviços e aos que podem ser afetados por sua escassez ${ }^{(1)}$.

A Bioética se propõe a refletir sobre a problemática, dos dilemas e desafios deste novo cenário, sugerindo condutas. Premissas éticas importantes precisam alicerçar as decisões e condutas, dos profissionais, mesmo diante da emergente situação. As principais premissas são: assegurar o respeito aos direitos humanos, dignidade e justiça à população. Entendendo que os desfechos de saúde da população, e em especial dos idosos, dependerão também dos profissionais que estarão a frente na assistência e/ou na tomada de decisão, estas premissas precisam ser consideradas.

Diariamente, as redes de notícias nacionais apresentam discordâncias entre os governos Federal, Estadual e Municipal, em relação a alocação dos recursos e estratégias de combate à pandemia, muitas vezes divergindo 
da orientação da Organização Mundial de Saúde (OMS). Essa discordância, associada às diversas realidades brasileiras, à escassez de recursos, às mudanças frequentes de diretrizes, e à falta de informações centralizadas, provocam inúmeros conflitos e inseguranças que refletem no atendimento a população.

A pandemia afeta diretamente os profissionais de saúde envolvidos, pois além de prestar atendimento a essa população, ficam mais expostos ao contágio, e, ainda precisam lidar com a escassez de recursos, a grande demanda de trabalho e o aumento da pressão frente à necessidade de apresentar respostas rápidas e eficazes quanto ao desfecho de saúde dos usuários do sistema de saúde.

A recomendação das Nações Unidas ${ }^{(1)}$ quanto às ações de assistência à saúde que afetam as pessoas idosas identifica quatro políticas de respostas imediatas e de longo prazo a se considerar na prática de saúde. Estas ações podem nortear uma reflexão bioética aos profissionais de saúde envolvidos no cuidado do idoso, e seguem:

1. Garantir que decisões difíceis de assistência à saúde que afetam as pessoas idosas sejam guiadas por um compromisso com a dignidade e o direito à saúde.

2. Fortalecer a inclusão social e a solidariedade durante o distanciamento físico.

3. Integrar totalmente o foco nas pessoas idosas na resposta socioeconômica e humanitária à COVID-19.

4. Expandir a participação de idosos, compartilhar boas práticas e aproveitar conhecimentos e dados ${ }^{(1)}$.

A garantia de que as decisões relacionadas ao uso dos recursos seja guiada pelas reais necessidades clínicas e baseadas em critérios éticos e evidências científicas incluindo a equidade no valor de vidas entre idosos e pessoas abaixo dos 60 anos, é um critério importante a ser considerado. As escolhas assertivas em meio à pandemia precisam ser inclusivas e, mesmo diante de protocolos e normas, a reflexão bioética feita pelos profissionais pode ser mais uma ferramenta usada em benefício da parte mais vulnerável da população em risco inclusive em relação à alocação de recursos.

O princípio da beneficência pode ser usado evitando o olhar voltado à capacidade de força de trabalho do indivíduo de forma utilitarista e garantindo, de certa forma, decisões éticas guiadas pela manutenção da dignidade.

O fortalecimento da inclusão social e da solidariedade durante o distanciamento físico da pessoa idosa pode ser observado sob olhar da autonomia na bioética. Com o compartilhamento de informações e consentimento esclarecido sobre técnicas e tratamentos disponíveis, a partir da supervisão do familiar ou cuidador (quando necessário), ou o respeito às diretivas antecipadas de vontade, o idoso tem o princípio da autonomia respeitado e, mesmo diante da emergência da situação e seguimento dos protocolos, o respeito à dignidade faz-se presente.

Ao integrar o foco nas pessoas idosas, na resposta socioeconômica e humanitária à COVID-19, dando ênfase ao cuidar e respeitando as particularidades de cada usuário do sistema sustenta-se o princípio da justiça. O idoso utiliza recursos materiais e humanos e não pode ser responsabilizado socioeconomicamente pela vulnerabilidade inerente. Evitando assim, o olhar utilitarista e adequando os cuidados a cada usuário de acordo com os recursos disponíveis.

A vulnerabilidade também faz-se presente entre os profissionais de saúde, que além de enfrentarem o risco da contaminação, lidam com as incertezas do novo cenário, cuja situação é emergencial, há escassez de recursos e possibilidade de realocação temporária de suas funções. É reconhecida a sobrecarga a que estão expostos. A ampliação das discussões e o diálogo com a equipe pode favorecer, no que tange à reflexões bioéticas, as decisões de alta complexidade auxiliando na tomada de decisão maximizando a ética e minimizando a tomada de decisão por conceitos morais particulares.

Assim, garantir os princípios éticos de respeito à integridade física e moral de todas as pessoas, às diferenças individuais, à justiça e à diversidade dos grupos sociais, com igualdade e equidade do idoso, são questões que necessitam de reflexão sob o prisma da bioética. Em casos críticos, onde a necessidade é maior que a 
disponibilidade de leitos e equipamentos é primordial a existência de recomendações brasileiras visando garantir o respeito aos princípios éticos no momento de "eleição" de qual paciente utilizará o recurso. Portanto, usar a idade cronológica como único critério para elegibilidade do paciente que receberá o atendimento é um critério que não respeita os princípios éticos.

A situação atual de pandemia pode ser uma oportunidade para mudanças positivas na sociedade, com vistas a ser mais inclusiva, equitativa e buscando cuidar da pessoa idosa com justiça e respeito (21).

\section{CONSIDERAÇÕES FINAIS}

É notável que em curto espaço de tempo os serviços de saúde, profissionais e usuários passaram por uma grande reorganização buscando soluções para as novas demandas que surgem com o cenário da pandemia mundial. A oferta de cuidados impacta diretamente os idosos, e os profissionais de saúde enfrentam decisões difíceis devido a escassez de recursos.

Tais fragilidades são expostas de forma mais contundente com a emergência da situação, a demanda de respostas rápidas exigidas devido ao quadro evolutivo da própria doença, os recursos escassos e o aumento do número de casos. Assim há possibilidades de erros que colocam em risco a segurança do paciente e expõem o paciente, o familiar, e a equipe assistencial envolvida.

Com o desafio de aumentar o número de leitos e a indispensável ampliação da equipe é esperado que nem todos os profissionais estejam treinados para atender nos diferentes cenários e usuários com necessidades específicas, como é o caso dos idosos.

Assim, a utilização de protocolos e normatizações associados a uma reflexão bioética possibilita aos profissionais tomadas de decisões de forma a assegurar que as necessidades individuais dos idosos sejam consideradas e respeitadas. Ou seja, não basta qualificação profissional e alocação adequada de recursos, é preciso que a equipe esteja qualificada para o cuidar, a fim de que esse seja realizado de forma justa.

Uma importante transição possivelmente esteja acontecendo na saúde, sendo importante avaliar os valores que prevalecem e os que precisam ser reforçados a fim de garantir tomadas de decisões justas. A reflexão bioética dos valores, ajustada aos esforços de seguir os protocolos é um instrumento eficaz na busca dos novos valores sociais.

\section{REFERÊNCIAS}

1. United Nations (UN). Policy Brief. The impactof COVID-19 on older persons. [Internet]. 2020 [cited 2020 Jun 10] Available from: https://unsdg.un.org/sites/default/files/2020-05/Policy-Brief-The-Impact-of-COVID-19-on-Older-Persons.pdf

2. World Health Organization (WHO). Preventing and managing COVID-19 across long-term care services: policy brief. [Internet]. 2020 [cited 2020 Jun 14]. Available from: https://www.who.int/publications/i/item/ WHO-2019-nCoV-Policy_Brief-Long-term_Care-2020.1

3. Ribeiro SCC. Alocação de recursos escassos em situações de catástrofe. In: Dadalto L. Bioética e COVID-19. Indaiatuba, São Paulo: Editora Foco; 2020.

4. Gurgel Jr GD, Leal EMM, Oliveira SRA, Santos FAS, Sousa IMC, Diderichsen F. Resource allocation for equity in Brazilian healthcare: a methodological model. Saúde Debate. 2019;43(121):329-40. https://doi.org/10.1590/0103-1104201912103

5. Barros MBA, Goldbaum M. Desafios do envelhecimento em contexto de desigualdade social. Suplemento ELSI-Brasil. Rev Saúde Pública. 2018;52(Suppl 2). https://doi.org/10.11606/S1518-8787.201805200supl2ed

6. Nunes BP, Souza ASS, Nogueira J, Andrade FB, Thumé E, Teixeira DSC, et al. Multimorbidade e população em risco para COVID-19 grave no Estudo Longitudinal da Saúde dos Idosos Brasileiros. Cad Saúde Pública. 2020;36(12):e00129620. https://doi.org/10.1590/0102-311x00129620

7. Hammerschmidt KSA, Santana RF. Saúde do idoso em tempos de pandemia Covid-19. Cogitare Enferm. 2020;25:e72849. http://dx.doi.org/10.5380/ce.v25i0.72849 
8. Associação Brasileira de Saúde Coletiva (ABRASCO). Frente pela vida. Plano nacional de enfrentamento à pandemia da COVID-19. ABRASCO: 2020. Available from: https://frentepelavida.org.br/uploads/documentos/PEP\%20COVID-19_V2.pdf

9. Beauchamp TL, Childress JF. Principles of biomedical ethics. 7.ed. New York: Oxford University Press, 2013.

10. Carmo ME, Guizardi FL. O conceito de vulnerabilidade e seus sentidos para as políticas públicas de saúde e assistência social. Cad Saúde Pública. 2018;34:e00101417. https://doi.org/10.1590/0102-311X00101417

11. Liu Z, Chen Y, Lin R, Han K. Clinical features of COVID-19 in elderly patients: a comparison with young and middle-aged patients. J Infect. 2020;80(suppl 6):e14-e18. https://doi.org/10.1016/j.jinf.2020.03.005

12. Shahid Z, Kalayanamitra R, McClafferty B, Kepko D, Ramgobin D, Patel R, et al. COVID -19 and Older Adults: what we know. J Am Geriatr Soc. 2020;68(suppl 5):926-9. https://doi.org/10.1111/jgs.16472

13. Pires LN, Carvalho L, Xavier LL. COVID-19 e desigualdade: a distribuição dos fatores de risco no Brasil. ONDAS, Universidade de Brasília (UnB). 2020. https://doi.org/10.13140/RG.2.2.27014.73282

14. Moraes EM, Viana LG, Resende LMH, Vasconcelos LS, Moura AS, Menezes A, Mansano NH, et al. COVID-19 nas instituições de longa permanência para idosos: estratégias de rastreamento laboratorial e prevenção da propagação da doença. Ciênc Saúde Coletiva. 2020;25(9):3445-58. https://doi.org/10.1590/1413-81232020259.20382020

15. Mendonça MHM, Silva Jr AGS, Cunha CLF, Latgé PK. A pandemia COVID-19 no Brasil: ecos e reflexos nas comunidades periféricas. APS Rev. 2020;2(suppl2):162-8. https://doi.org/10.14295/aps.v2i2.124

16. Kalache A, Silva A, Giacomin KC, Lima KC, Ramos LR, Louvison M, et al. Envelhecimento e desigualdades: políticas de proteção social aos idosos em função da Pandemia Covid-19 no Brasil. Rev Bras Geriatr Gerontol. 2020;23(suppl6):e200122. https://doi.org/10.1590/1981-22562020023.200122

17. Organização das Nações Unidas para a Educação, a Ciência e a Cultura (UNESCO). Recomendaciones para la toma de decisiones éticas sobre el acceso de pacientes a unidades de cuidados especiales en situaciones de pandemia[Internet]. 2020 [cited 2020 Jun 14]. Available from: http://www.bioeticayderecho.ub.edu/sites/default/files/doc_recom-pandemia.pdf

18. Rene R, Barnes-Kentish N, Boyer A, Laurent A, Azoulay E, Reignier J. Ethical dilemmas due to the Covid-19 pandemic. Ann Intensive Care. 2020,10(suppl1):1-9. https://doi.org/10.1186/s13613-020-00702-7

19. Associação de Medicina Intensiva Brasileira (AMIB). Associação Brasileira de Medicina de Emergência (ABRAMEDE). Sociedade Brasileira de Geriatria e Gerontologia (SBGG). Academia Nacional de Cuidados Paliativos (ANCP). Recomendações da AMIB, ABRAMEDE, SBGG e ANCP de alocação de recursos em esgotamento durante a pandemia por COVID-19[Internet]. 2020 [cited 2020 Jun 10]. Available from: https://www.amib.org.br/noticia/nid/recomendacoes-daamib-abramede-sbgg-e-ancp-de-alocacao- de-recursos-em-esgotamento-durante-a-pandemia-por-covid-19

20. Observatório de Direitos dos Pacientes. Sociedade Brasileira para a Qualidade do Cuidado e Segurança do Paciente (SOBRASP). Diretrizes para a alocação de recursos de cuidados intensivos no contexto da COVID-19[Internet]. 2020 [cited 2020 Jun 11]. Available from: https://www.sobrasp.org.br/post/ diretrizes-para-a-alocacao-de-recursos-de-cuidados-intensivos-no-contexto-da-covid19

21. Mulinari F. Ética e Justiça Social em tempos de pandemia. Rev Int Filos. 2020:11:e42. https://hcommons.org/deposits/item/hc 